\title{
Propuesta de integración del modelo para construcción de soluciones y el modelo de negocio Canvas para el desarrollo de sistemas telemáticos*
}

\author{
Diego Fernando Burbano Santacruz \\ Eduardo Rojas Pineda***
}

Recibido: 07/09/2015 - Aceptado: 27/10/2016

DOl: 10.22395/rium.v16n31a9

\begin{abstract}
Resumen
Este artículo, resultado de investigación, propone un marco de referencia para el uso del Lienzo del Modelo de Negocios Canvas (LMN) en conjunto con el Modelo de Construcción de Soluciones (MCS) para el desarrollo de sistemas telemáticos. A través de la utilización del LMN y su traducción para el modelado del negocio basado en UML y las directrices del Proceso Unificado de Rational (RUP), se facilita el trabajo de entendimiento del contexto organizacional donde operará la solución y el diálogo entre los interesados. Opcionalmente se propone utilizar un producto mínimo viable (PMV), según lo define el Lean Startup, como complemento a la idea de ejercitar el escenario de solución antes de pasar a las fases de construcción. El marco de referencia fue puesto a prueba con el desarrollo de una solución telemática para la Facultad de Ciencias de la Salud de la Universidad del Cauca, mostrando sus bondades y facilitando a los desarrolladores y clientes un mejor entendimiento del contexto organizacional y de la evolución y definición de la solución a construir.
\end{abstract}

Palabras clave: desarrollo de sistemas telemáticos; desarrollo de software; generación de modelos de negocio; modelo de negocio Canvas; modelo para construcción de soluciones.

Artículo derivado del proyecto de investigación Propuesta de integración del modelo de negocio Canvas (MNC) y el modelo para construcción de soluciones (MCS) para el desarrollo de sistemas telemáticos. Fechas de realización del proyecto: 20 de marzo de 2015 al 7 de octubre de 2015. Entidades financiadoras: Departamento de Telemática, Facultad de Ingeniería Electrónica y Telecomunicaciones, Universidad del Cauca, Popayán, Colombia.

** Ingeniero en Electrónica y Telecomunicaciones, Grupo de Ingeniería Telemática, Universidad del Cauca, Popayán, Colombia. Carrera 2 \#18N-61, casa 5, Portales de Pomona. Teléfono: $+57+2838$ 2895. diegoburbano@ unicauca.edu.co

*** M. Sc. en Sistemas y Redes de Comunicaciones. M. Sc. en Dirección Universitaria. Profesor de tiempo completo Departamento de Telemática, Grupo de Ingeniería Telemática, Universidad del Cauca, Popayán, Colombia. Edificio Ingenierías, Tulcán, Oficina 404, Teléfono: +57+2820 9800, ext. 2175. erojas@unicauca.edu.co 


\title{
Integration proposal of the model for the construction of solutions and the Canvas business model for the development of telematic systems
}

\begin{abstract}
This article is the result of a research. It proposes a reference framework for using the Canvas Business Model in conjunction with the Solutions Construction Model for the development of telematics systems. By using the CBM and its translation for the modeling of UML-based business and the Rational Unified Process (RUP) guidelines, understanding process of the organizational context where the solution will operate and dialogue between the interested parties becomes easier. Optionally, the use of the Minimum Viable Product (MVP) is proposed, as defined in the Lean Startup, as complement to the idea of operating the solution scenario before going forward to the construction stages. The reference framework was tested with the development of a telematics solution for the School of Health Sciences of Universidad del Cauca, showing its advantages and facilitating developers and customers a better understanding of the organizational context and the evolution and definition of the solution to be constructed.
\end{abstract}

Keywords: telematics systems development; software development; generation of business models; Canvas Business Model; solutions construction model.

\section{Proposta de integração do modelo para a construção de soluções e o modelo de negócio Canvas para o desenvolvimento de sistemas telemáticos}

\begin{abstract}
Resumo
Este artigo, resultado de pesquisa, propõe um referencial para o uso do Quadro de Modelo de Negócios (BMC, Business Model Canvas) em conjunto com o Modelo de Construção de Soluções (MCS) para o desenvolvimento de sistemas telemáticos. Por meio da utilização do BMC e de sua tradução para o modelo de negócio baseado em UML e nas diretrizes do Processo Unificado da Rational (RUP, por sua sigla em inglês), facilita-se o trabalho de entendimento do contexto organizacional em que operarão a solução e o diálogo entre os interessados. Como opcional, propõe-se utilizar um Produto Mínimo Viável (MVP, por sua sigla em inglês), segundo o define Lean Startup, como complementação à ideia de exercitar o cenário de solução antes de passar às fases de construção. O referencial foi testado com o desenvolvimento de uma solução telemática para a Faculdade de Ciências da Saúde da Universidad del Cauca (Colômbia), mostrando seus benefícios e facilitando aos desenvolvedores e clientes um melhor entendimento do contexto organizacional e da evolução e definição da solução a construir.

Palavras-chave: desenvolvimento de sistemas telemáticos; desenvolvimento de software; geração de modelos de negócio; Business Model Canvas; modelo para a construção de soluções.
\end{abstract}




\section{INTRODUCCIÓN}

El Modelo para Construcción de Soluciones (MCS) [1], desarrollado por el Departamento de Telemática de la Universidad del Cauca, establece una referencia metodológica esencial para cualquier proyecto que tenga como propósito construir una solución basada en un sistema telemático, de calidad, oportuna y con costos competitivos.

Por otro lado, Alexander Osterwalder, junto a su director Yves Pigneur, propuso en su tesis de doctorado, una metodología que permite describir un modelo de negocio teniendo en cuenta 9 aspectos esenciales que definen la lógica que toda organización debería seguir para crear, proporcionar y capturar valor [2]

Este trabajo plantea la posibilidad de establecer un ambiente, que de manera integrada, aborde los aspectos del modelo de negocio de una organización y sus estrategias asociadas, con la descripción y desarrollo del sistema telemático que configura la propuesta de valor sobre la cual satisfacer las necesidades o expectativas de los clientes o usuarios de dicha organización.

Para la elaboración de la presente investigación se utilizó como referencia metodológica el modelo de investigación documental descrito en [3] y una instanciación de la integración de MCS y el Lienzo del Modelo de Negocio Canvas (LMN) para la construcción del ejemplo de aplicación.

\section{MATERIALES Y MÉTODOS}

La propuesta de integración entre el MCS y el LMN para el desarrollo de sistemas telemáticos pretende mejorar la descripción del modelo de negocio. Para ello es necesario definir el contexto en el cual es trabajado el concepto de modelado del negocio.

\subsection{Modelado del negocio}

En el contexto de los sistemas telemáticos, el modelado del negocio se define como el conjunto de técnicas y artefactos que actúan como guía necesaria para visualizar y entender los procesos de negocio, realizando una descripción operacional de una determinada organización o empresa, y obtener como producto el modelo del negocio. En ese orden de ideas un modelo de negocio se hace necesario en al menos una de tres situaciones [3]:

- Reingeniería del negocio. Implica analizar y fundamentalmente repensar cómo opera el negocio y cómo se articula con el mundo exterior.

- Mejorar los procesos del negocio. Una reingeniería limitada a un subconjunto de los procesos del negocio para mejorar su competitividad. 
- Automatizar los procesos del negocio. Reducir los recursos requeridos por un proceso, limitando la intervención humana.

Una cuarta podría ser, incluso, la creación de un nuevo negocio; esta connotación está en principio asociada al modelo orientado a la propuesta de valor y al concepto de cliente citado por Osterwalder en [4], para lo cual es necesario adoptar algunos conceptos referentes a emprendimiento que complementarán el marco teórico, logrando con ello identificar la posibilidad de integrar las directrices de RUP [5] y el lenguaje UML [6] con las del modelo de negocio basado en ontologías, formalizado inicialmente en [7], complementado en [8] y dado a conocer internacionalmente en [2].

\subsection{EL MCS Y EL MODELADO DEL NEGOCIO}

Tanto en RUP como en el MCS, el modelo del proceso de desarrollo se caracteriza por ser iterativo e incremental. El proyecto es dividido en iteraciones o mini proyectos que van generando un incremento en el sistema, siguiendo un esquema en espiral sobre 4 fases (figura 1): estudio de pre-factibilidad, formulación del proyecto, ejecución del proyecto y validación de la solución [1]. Como resultado de la ejecución de las diferentes fases, lo que se obtiene es un conjunto de representaciones del sistema solución con diferentes niveles de abstracción, los cuales se describen en la tabla 1.

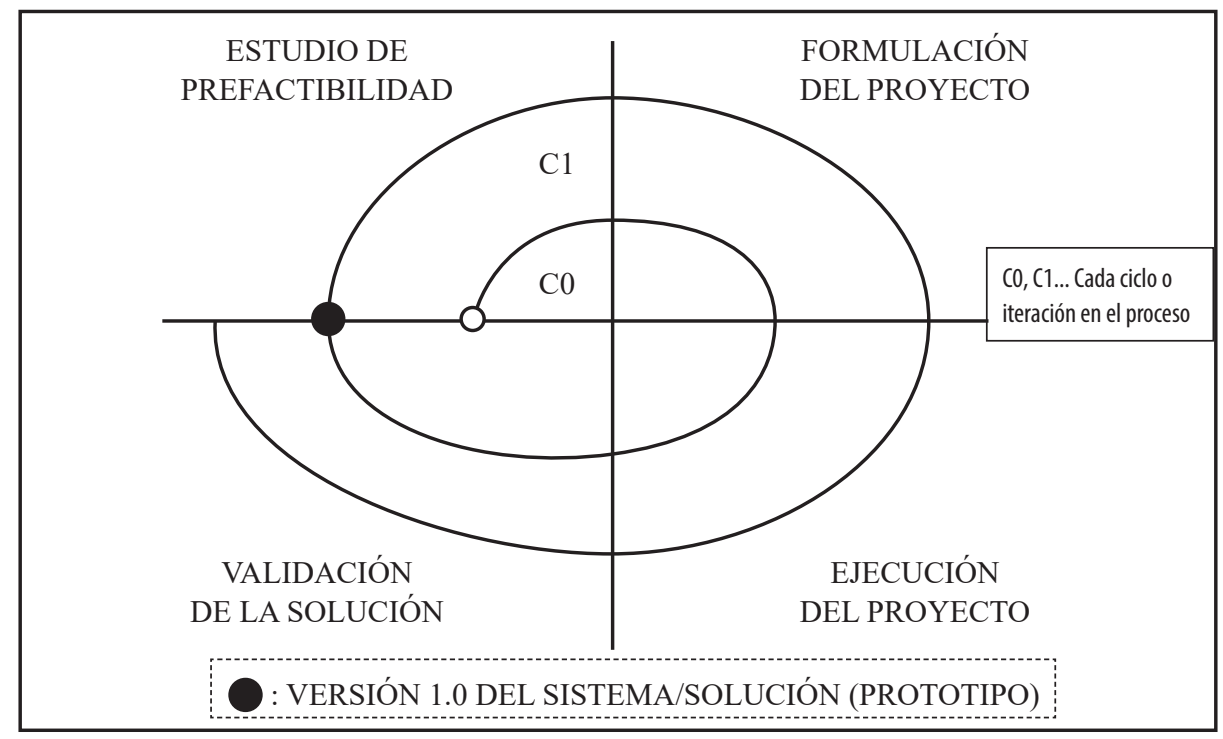

Figura 1: visión general del Modelo de Proceso de Desarrollo del MCS Fuente: [1] 
Tabla 1: estructura de referencia para la descripción del sistema/solución

\begin{tabular}{|c|c|}
\hline Macrocomponentes & Componentes principales \\
\hline $\begin{array}{l}\text { Modelo para } \\
\text { establecimiento de } \\
\text { responsabilidades }\end{array}$ & $\begin{array}{l}\text { 1. Modelo del Ambiente del Sistema } \\
\text { 2. Modelo de Casos de Uso del Sistema (Esencial) } \\
\text { 3. Arquitectura de Referencia para el Sistema/Solución (Esencial) } \\
\text { 4. Registro de Gestión de Riesgos } \\
\text { 5. Plan del Proyecto (Esencial) } \\
\text { 6. Caso del Negocio }\end{array}$ \\
\hline $\begin{array}{l}\text { Modelo para descripción } \\
\text { del sistema }\end{array}$ & $\begin{array}{l}\text { 7. Modelo del Diseño del Sistema/Solución (Esencial) } \\
\text { 8. Modelo de despliegue/Distribución del Sistema/Solución } \\
\text { 9. Modelo de Pruebas }\end{array}$ \\
\hline $\begin{array}{l}\text { Modelo de implementación } \\
\text { del sistema }\end{array}$ & $\begin{array}{l}\text { 10. Descripción detallada del Sistema/Solución } \\
\text { 11. Prototipo Operacional de la Solución versión beta (Esencial) } \\
\text { 12. Manuales de Usuario e información complementaria. }\end{array}$ \\
\hline
\end{tabular}

Fuente: [1]

- Estudio de pre-factibilidad: su propósito es analizar el dominio del problema a través de un modelo del negocio de la organización, y determinar si es viable continuar con el proyecto. Genera un modelo de establecimiento de responsabilidades inicial que describe el alcance del sistema solución.

- Formulación del proyecto: su propósito es analizar los aspectos esenciales relacionados con la construcción del sistema solución, asegurando su viabilidad. Genera un modelo de establecimiento de responsabilidades final que describe la arquitectura de referencia que soporta el proceso de desarrollo del sistema solución.

- Ejecución del proyecto: su propósito es construir incrementalmente las versiones correspondientes del sistema solución. Genera los modelos para descripción del sistema y los modelos para implementación del sistema correspondientes a la capacidad operacional que se haya proyectado.

- Validación de la solución: su propósito es ejercitar el sistema solución detectando y corrigiendo posibles fallas hasta lograr la satisfacción del cliente con el sistema solución.

Las cuatro fases establecidas en el MCS mantienen una misma estructura de referencia, la cual comprende cuatro actividades: revisión y compromiso, en donde se revisan las condiciones del proyecto y definen los compromisos de todos los involu- 
crados; planeación, en donde se define la manera en que se llevará acabo el trabajo de dicha fase; flujo de trabajo, que representa una iteración a través de la cual se integran dos o más de las actividades de captura de requerimientos, análisis, diseño, implementación y prueba de los componentes del sistema solución; evaluación de la fase, en donde se establece el grado de cumplimiento de los compromisos y se define si es viable proceder a una fase posterior o realizar una nueva iteración.

El interés de este trabajo está enfocado en la fase del estudio de prefactibilidad, debido a que en ella se utiliza un modelo de negocio de la organización como herramienta para entender el problema y generar un primer modelo de la solución a alto nivel de abstracción. El MCS adopta los estereotipos definidos en UML para la identificación de los elementos del modelado del negocio (ver tabla 2).

Tabla 2: estereotipos del Modelado del Negocio en UML

\begin{tabular}{|c|c|}
\hline Actor del Negocio & Objetivo del negocio \\
\hline Trabajador del Negocio & Entidad del negocio \\
\hline Evento del Negocio & Regla del Negocio \\
\hline Caso de Uso del Negocio (CUN) & \\
\hline
\end{tabular}

Fuente: elaboración propia.

El MCS propone para el modelado del negocio los modelos basados en UML: modelo de casos de uso del negocio (MCUN) y modelo de análisis del negocio (MAN) (tabla 3). Estos modelos constituyen un requisito esencial en el estudio de prefactibilidad del MCS para el entendimiento del negocio del cliente y, por ende, para el planteamiento inicial del sistema solución. Es aquí donde la utilización del LMN puede enriquecer el proceso de descripción del negocio y facilitar el entendimiento con el cliente. 
Tabla 3: modelos para el modelado del negocio en UML

\begin{tabular}{|c|c|}
\hline Modelo de Casos de Uso del Negocio (MCUN) & $\begin{array}{l}\text { Describe la forma en que una empresa } \\
\text { interactúa con su entorno, mediante la } \\
\text { declaración de los procesos de negocio } \\
\text { representados como Casos de Uso y } \\
\text { el cómo se beneficia e interactúan los } \\
\text { clientes o socios en dichos procesos. }\end{array}$ \\
\hline $\begin{array}{c}\text { Business Analysis } \\
\text { Model } \\
\text { Business Worker Classes }\end{array}$ & $\begin{array}{l}\text { Corresponde a la vista lógica del mode- } \\
\text { lado. Se encarga de modelar la interac- } \\
\text { ción entre trabajadores del negocio y } \\
\text { entidades del negocio. En el MCS este } \\
\text { modelo se conoce como: Modelo de } \\
\text { Objetos del Negocio. }\end{array}$ \\
\hline Modelo de Análisis del Negocio (MAN) & \\
\hline
\end{tabular}

Fuente: Elaboración propia.

\subsection{Generación de modelos de negocio}

La propuesta para generar modelos de negocios de Osterwalder [2] establece cinco fases claramente definidas en el proceso de diseño del modelo de negocio -PDMN- y propone un LMN en donde son integrados los 9 bloques que comprende el modelo de negocio.

\subsubsection{Fases del proceso de diseño del modelo de negocio}

Las cinco fases propuestas por Osterwalder [2] para la generación del modelo de negocio se pueden describir de la siguiente manera:

- Movilización: consiste en la preparación de un proyecto para el diseño del modelo de negocio.

- Comprensión: consiste en recabar información pertinente respecto a los clientes, la tecnología y el entorno.

- Diseño: comprende la adaptación y modificación del modelo de negocio.

- Aplicación: configura la puesta en marcha del modelo de negocio.

- Gestión: comprende la supervisión, evaluación o transformación del modelo de negocio. 


\subsubsection{Herramientas y técnicas en el ámbito del PDMN}

A lo largo de las diferentes fases propuestas para el PDMN por Osterwalder en [2] se recomienda la utilización de varias de las herramientas o técnicas que se describen a continuación:

a. Lienzo del modelo de negocio (LMN): herramienta que constituye el medio de comunicación (idioma) común para el diseño del modelo de negocio.

b. Narración de historias: técnica que ayuda a describir eficazmente el modelo de negocio y a incentivar un debate en profundidad del mismo.

c. Patrones de modelo de negocio: en [2] se describen 5 tipos de modelos de negocio a manera de arquetipos reutilizables que facilitan la comprensión del modelo propio.

d. Aportaciones de clientes: el conjunto de técnicas que facilitan la interacción con las necesidades reales de los clientes potenciales.

e. Pensamiento visual: técnica que se refiere al uso de elementos visuales que faciliten una mirada comprensiva del modelo de negocio (caricaturas, adhesivos, diagramas, etc.).

f. Escenarios: técnica propuesta para evaluar posibles escenarios, que permite prever con anticipación el entorno en el que un modelo de negocio competirá en el futuro o plantear alternativas con relación al comportamiento de los clientes.

g. Entorno del modelo de negocio: técnica cuyo propósito es comprender mejor las principales fuerzas externas que influirán sobre el modelo de negocio, esbozando el comportamiento de las fuerzas del mercado, las fuerzas de la industria, las tendencias clave y las fuerzas macroeconómicas.

h. Evaluación de modelos de negocio: actividad de gestión que provee los medios para una mejora continua del modelo de negocios, basado en un análisis general de la evolución estratégica del modelo o en una revisión detallada de debilidades, fortalezas, oportunidades y amenazas (DOFA) del modelo de negocio y de cada.

i. Ideación: técnica que consiste en la generación de ideas para el diseño de modelos de negocio a partir de las tendencias del mercado futuro.

j. Creación de prototipos: herramienta que tiene como objetivo el debate, el análisis y la corrección de un concepto, asociado a un determinado modelo de negocio. En el Lean Startup se opera a través del producto mínimo viable (PMV).

k. Perspectiva de los modelos de negocio mediante la estrategia del océano azul: 
técnica que propone centrarse en la creación de modelos de negocio innovadores basados en la diferenciación fundamental. Su contraparte es la estrategia del océano rojo, donde los esfuerzos son orientados a mejorar la capacidad de captar clientes en entornos de alta competencia.

1. Gestión de varios modelos de negocio: técnica que facilita el definir la forma de gestionar nuevos modelos de negocio en entornos que ya tienen algunos en funcionamiento.

La tabla 4 relaciona la influencia de las técnicas y herramientas anteriores sobre cada fase del PDMN, sugiriendo también una secuencia en el uso de cada una.

Tabla 4: influencia de las técnicas o herramientas en las fases del PDMN

\begin{tabular}{|c|c|c|c|c|c|}
\hline Fases & Movilización & Comprensión & Diseño & Aplicación & Gestión \\
\hline $\begin{array}{c}\text { Técnicas o } \\
\text { herramientas }\end{array}$ & $\mathrm{a}, \mathrm{b}$ & $\mathrm{a}, \mathrm{c}, \mathrm{d}, \mathrm{e}, \mathrm{f}, \mathrm{g}, \mathrm{h}$ & $\begin{array}{c}\mathrm{a}, \mathrm{c}, \mathrm{i}, \mathrm{e}, \mathrm{j}, \mathrm{f}, \\
\mathrm{h}, \mathrm{k}, \mathrm{l}\end{array}$ & $\mathrm{a}, \mathrm{e}, \mathrm{b}, \mathrm{l}$ & $\mathrm{a}, \mathrm{e}, \mathrm{f}, \mathrm{g}$, \\
\hline
\end{tabular}

Fuente: elaboración propia.

En la tabla 4, se observa que el LMN es común en cada fase del PDMN; esto debido a que el autor propone el uso de un lienzo para definir nueve bloques temáticos, los cuales agrupan las principales variables objeto del PDMN.

\subsubsection{Lienzo del modelo de negocio (LMN)}

El LMN está compuesto por 9 módulos descritos a continuación según las definiciones dadas por Osterwalder en [8] (ver figura 2):

- Segmentos del mercado (SM): describe un segmento de clientes objetivo que define el tipo de clientes que una empresa quiere alcanzar.

- Propuestas de valor (PV): una propuesta de valor representa un valor para uno o varios cliente(s) objetivo y se basa en una o varias capacidad(es). Se puede descomponer en un conjunto de ofertas elementales. Una propuesta de valor se caracteriza por sus atributos descripción, razones, nivel de valor y nivel de precio, y opcionalmente ciclo de vida.

- Canales (C): un canal de distribución describe cómo una compañía entrega una propuesta de valor a un segmento de clientes objetivo. Normalmente una empresa dispone de uno o varios canal(es) directos o indirectos que se pueden descomponer en su enlace(es). 


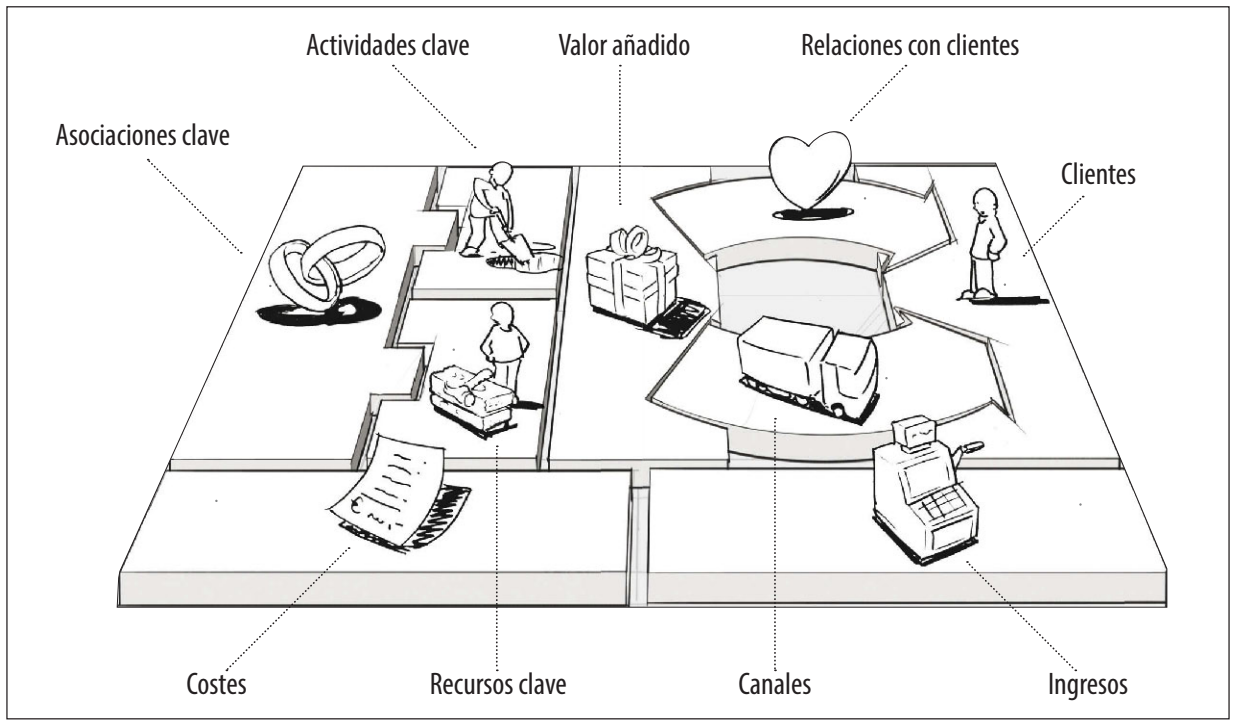

Figura 2: bloques o módulos del LMN Fuente: [2]

- Relaciones con clientes (RCI): el elemento relación describe el vínculo que una empresa establece con un segmento de clientes objetivo. Una relación se basa en la importancia del cliente y se puede descomponer en varios mecanismos de relación.

- Fuentes de ingresos (FI): el modelo de ingresos describe la forma en que la compañía hace dinero. Puede estar compuesto por uno o varios elementos del flujo de ingresos y precios.

- Recursos clave (RC): describe la capacidad de ejecutar un patrón repetible de acciones. Una empresa tiene que disponer de una serie de capacidades para poder ofrecer su propuesta de valor. Las capacidades se basan en un conjunto de RECURSOS de la empresa o de su socio(s). Los recursos son los insumos en el proceso de creación de valor. Ellos son la fuente de las capacidades que una empresa necesita con el fin de proporcionar sus propuestas de valor.

- Actividades clave (AC): la configuración del valor de una empresa describe la disposición de una o varias actividad(es) con el fin de ofrecer una propuesta de valor. Una actividad es una acción que una empresa lleva a cabo para hacer negocios y lograr sus objetivos.

- Asociaciones clave (AsC): una asociación es un acuerdo de cooperación iniciado, voluntariamente formado entre dos o más empresas independientes, con el fin de 
llevar a cabo un determinado proyecto o actividad en forma conjunta mediante la coordinación de las capacidades necesarias, recursos y actividades.

- Estructura de costos (EC): el elemento estructura de costos mide todos los costos monetarios en que incurre la empresa.

\subsection{Lean Startup (Producto Mínimo Viable (PMV))}

El método Lean StartUp [8] está enfocado en la construcción de una empresa por medio del desarrollo de clientes [9]. Tal concepto aclara que es necesario realizar una realimentación (feedback) con el cliente para lo cual se propone utilizar un producto mínimo viable (PMV).

Una de las reglas de introducir un PMV para el aprendizaje del mercado es hacerlo por medio de iteraciones, con las cuales lograremos extraer conocimiento desde el cliente, mediante una serie de intentos o pivotes sobre el mismo PMV, que constituyen el bucle construir-medir-aprender (figura 3).

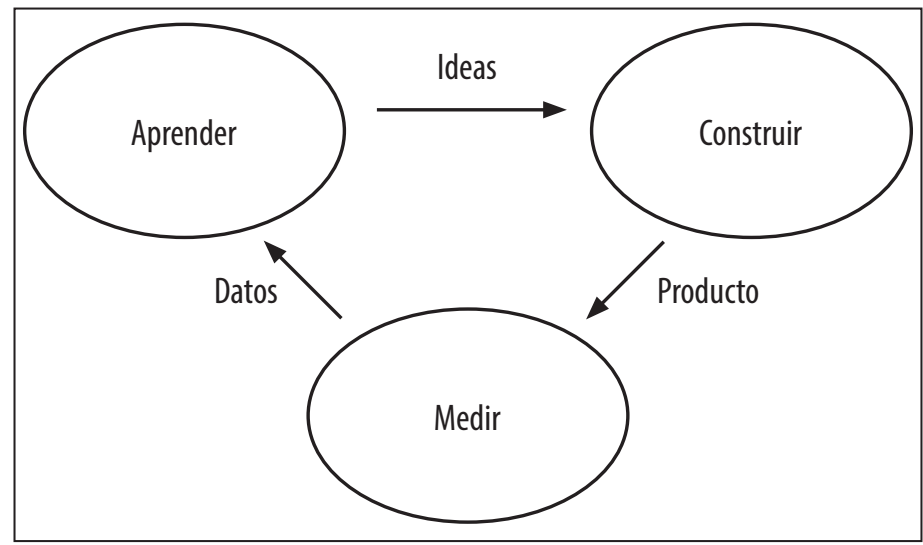

Figura 3: bucle construir-medir-aprender del PMV

Fuente: [8]

El objetivo es identificar el camino para encontrar una solución adecuada a un problema por el cual los primeros clientes ("early adopters") que adopten la solución estarían dispuestos a pagar. Generalmente, estas soluciones son expuestas de diferente forma; aquellas más comunes citadas en [10] son:

- Test de humo: por medio de publicidad, llevar al cliente a un sitio donde es notificado del estado en desarrollo del sistema solución.

- Power point: presentación con las características del producto, sin necesidad de realizar una versión preliminar. 
- Video: equivalente a la anterior, pero usando un medio audiovisual.

- Maqueta: Modelo a escala de la solución final.

- Prototipo: versión artesanal, con mínima implementación y puntual de lo que será el producto final.

- Mago de $O z$ : presentación de la fachada de la solución final sin desarrollo tras bambalinas.

\section{PROPUESTA DE INTEGRACIÓN MCS-LMN}

Los sistemas objetivo a los que hace referencia este artículo son los sistemas de aplicación telemáticos orientados a la solución de un problema específico.

Uno de los aspectos que trabaja el estudio de prefactibilidad del MCS es precisamente la declaración inicial del negocio, en donde será descrito el contexto en el que funcionará el sistema solución. Una vez se tiene claro el funcionamiento interno de una organización, se podrán identificar los problemas que esta posee en la realización de sus procesos de negocio. Esta actividad requiere de la construcción del MCUN y el MAN, pero el MCS no prescribe herramienta alguna que facilite abordar el entendimiento del ambiente del problema, más allá de establecer contacto con el cliente. Por lo tanto, esta propuesta de integración contribuirá con un marco referencial aún no existente en el MCS para facilitar la comunicación con el cliente y construir los modelos del negocio en UML. Permitirá mejorar la descripción del modelo de negocio, a través de los siguientes propósitos:

- Comprender la estructura y la dinámica de la organización.

- Comprender los problemas actuales de la organización, identificar potencial de crecimiento y mejoras.

- Asegurar el total entendimiento entre los clientes / usuarios y desarrolladores, facilitando un medio de entendimiento común entre las partes.

- Propiciar las facilidades para la derivación de los requerimientos del sistema, necesarios para la creación del sistema / solución.

\subsection{Marco de referencia}

El diagrama de la figura 4 describe la arquitectura modular de la propuesta de integración.

Según la figura 4, el cliente y la descripción de su negocio proporcionarán un conjunto de conceptos y términos que se deben incluir en el glosario del negocio (GN), 
el objetivo del negocio $(\mathrm{ON})$ y las reglas del negocio $(\mathrm{RN})$. Estos se utilizarán para obtener el LMN de la organización (modelo del negocio del cliente). A partir de este se obtienen los modelos MCUN y MAN que describen, en términos de lenguaje UML, el modelo de negocio de la organización siguiendo los lineamientos del MCS. De manera opcional, y a partir del LMN del sistema / solución (modelo de la solución), es posible crear y socializar un PMV que facilite la validación del aprendizaje.

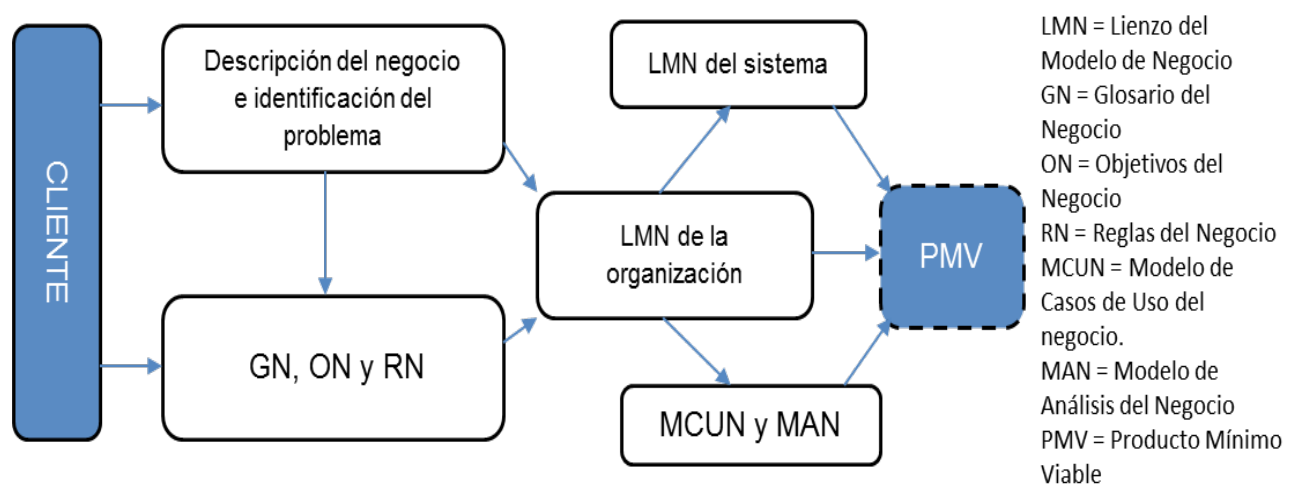

Figura 4: Arquitectura de referencia de la propuesta.

Fuente: elaboración propia

\subsubsection{Descripción del negocio del cliente e identificación del problema}

Entender la estructura y dinámica de una organización permitirá identificar los problemas que esta posee, las potenciales mejoras y asegurar el entendimiento común entre sus integrantes y el equipo de desarrollo que abordará la solución.

\subsubsection{Obtención del GN, ON y RN}

El modelo genérico para el modelado del negocio explicitado en [11] propone que para construir los modelos MCUN y MAN es necesario contar, como mínimo, con los artefactos GN, ON y RN. Cada uno de estos artefactos posee una relación única respecto al MCU, como se observa en la figura 5. 


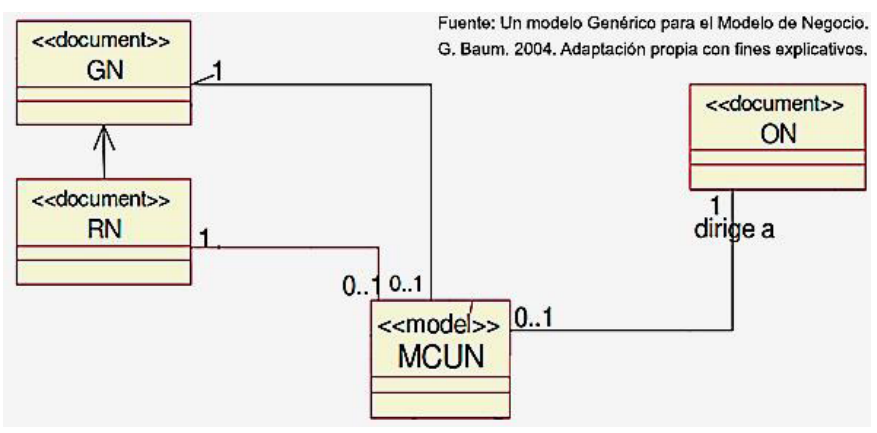

Figura 5: relación del GN, ON y RN con el MCUN Fuente:[11]

\subsubsection{LMN de la organización y derivación del MCUN y del MAN}

El mecanismo de comunicación no ambigua, para el entendimiento común de todos los involucrados en el desarrollo de un sistema / solución, corresponde al LMN, facilitando el diálogo con el cliente y la identificación de los elementos necesarios para la construcción del MCUN y el MAN.

Teniendo en cuenta la información correspondiente al marco conceptual del MNC es posible proponer que cada módulo del LMN comprenda un conjunto de estereotipos y artefactos específicos en cada uno de los módulos, como lo ilustra la figura 6 .

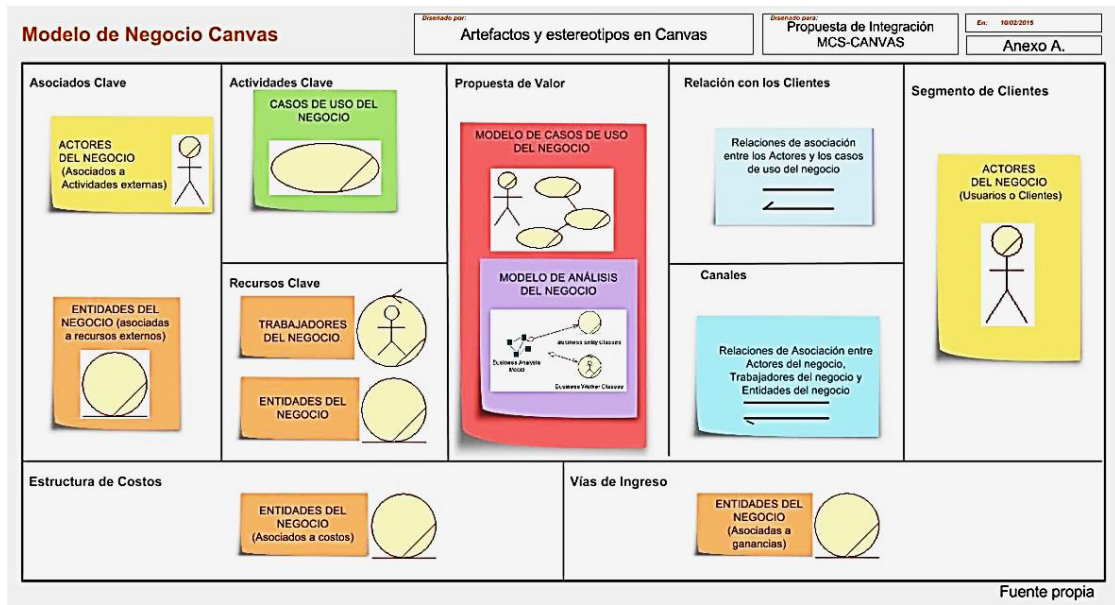

Figura 6: artefactos y estereotipos del Modelo del Negocio en el LMN.

Fuente: elaboración propia

A partir de la definición y descripción de los 9 componentes del LMN dada por Osterwalder en [8] y esbozadas en el aparte 1.3.3 de este documento, es posible iden- 
tificar los distintos elementos requeridos para construir el MCUN y el MAN, descritos con UML. Así tenemos que el módulo "Actividades Clave" del LMN constituye una abstracción de cada uno de los procesos, compuestos por una o más actividades, que realiza la organización para ofrecer su propuesta de valor, configurando cada uno de ellos un servicio o caso de uso en términos de los diagramas de UML. Las actividades clave son desarrolladas por uno o varios actores propios de la organización o provenientes de los asociados clave y se relacionan con unos recursos propios o de los asociados clave, permitiendo identificar en los módulos del LMN "Recursos Claves" y "Asociados Clave", los trabajadores del negocio y las entidades del negocio $\mathrm{y}$, posiblemente, otros actores del negocio, en términos de los diagramas de UML. El módulo de la "Estructura de Costos", aunque se concentra en describir los costos en que incurre la organización, es posible que permita identificar otras entidades del negocio en términos de UML, que no estén directamente involucradas en las actividades clave.

Por otro lado, el módulo de "Segmentos del mercado" del LMN claramente permite identificar los clientes o usuarios del negocio, actores del negocio en términos de los diagramas de UML. Los módulos de "Relación con los clientes" y "Canales", describen las formas en que se entrega el valor a los clientes o usuarios, permitiendo identificar cómo estos se asocian con cada uno de los casos de uso que componen la propuesta de valor y con los trabajadores o entidades del negocio a través de interfaces bien establecidas, en términos de los diagramas de UML. El módulo "Fuentes de ingreso" puede permitir la identificación de entidades del negocio adicionales en términos de UML, relacionadas con las estrategias de ingreso definidas para el modelo del negocio.

Finalmente, el módulo "propuesta de valor" del LMN es una descripción general del conjunto de los productos y servicios de una empresa que son de valor para el cliente. En términos de la representación en UML, dicha descripción debe corresponder con los diagramas del MCUN y con el MAN, facilitando identificar el núcleo principal en el que se basa la propuesta de valor.

\subsubsection{LMN inicial para la construcción del sistema}

El LMN deberá servir de soporte para el cumplimiento de los objetivos y etapas de referencia de la primera fase del proceso de desarrollo del MCS, cuyo objetivo general consiste en "Declarar el alcance del sistema que se quiere crear y establecer si es viable iniciar el respectivo proyecto" [1, p. 17]. A continuación, se propone un conjunto de preguntas clave para cada módulo del LMN que describe el sistema solución.

- Asociaciones clave: ¿A quién o a qué se acude para poder hacer posible la creación del sistema / solución? 
- Actividades clave: además de realizar actividades asociadas a la factibilidad del proyecto, ¿qué actividades clave son necesarias para la creación de una solución acorde con los requisitos del cliente?

- Recursos clave: además del recurso humano medido en tiempo y esfuerzo, ¿qué otros recursos clave son indispensables para el desarrollo de la solución?

- Estructura de costos: ¿cuánto cuestan los recursos clave, las actividades clave y mantener a los asociados clave?

- Segmento de clientes: ¿para quién o para quienes se construye la solución?

- Relación con los clientes: ¿de qué maneras podemos crear o mantener las relaciones que nos permitan retener o adquirir los clientes?

- Canales: ¿cómo es posible hacer asequible el sistema/ solución al cliente? ¿Cuáles son los medios que el cliente utilizará para interactuar con la solución?

- Vías de ingreso: ¿cuánto cuesta el trabajo del equipo del proyecto? ¿Respecto a qué criterios basados en la estructura de costos, es posible cobrar más o cobrar menos?

- Propuesta de valor: debemos procurar que el cliente se haga a una: "Solución de calidad, oportuna y con costos competitivos”. ¿Nombre de la solución?

\subsubsection{Creación y socialización de un PMV}

Para utilizar un PMV se plantean las siguientes recomendaciones:

- Seleccionar el caso de uso de negocio con base en el procedimiento que se considere más representativo.

- Crear: seleccionar una estrategia mediante la cual sea posible dar una mínima funcionalidad al cliente.

- Medir: obtener datos detallados sobre la reacción del cliente respecto a la propuesta realizada.

- Aprender: captar toda la información recolectada y sacar conclusiones.

- Si el producto es aceptado constituirá un argumento a favor del modelo de negocio previamente diseñado; en caso contrario, deberá identificarse por qué el sistema solución no cubre las expectativas y repetir el proceso.

Finalmente, es posible afirmar que el PMV es un complemento para el estudio de pre-factibilidad, en especial en la declaración de la viabilidad de la arquitectura inicial, puesto que "el propósito de plantear una arquitectura inicial es permitir tener cierto grado de confianza en la viabilidad de implementar el sistema especificado" [1, p. 20], como también en la identificación de riesgos críticos y su mitigación. 


\title{
3. RESULTADOS Y DISCUSIÓN
}

La creación de la "plataforma para la gestión de la información referente a las Prácticas de Formación en Salud de los estudiantes de la Facultad de Ciencias de la Salud de la Universidad del Cauca-GestRotes" utilizó como referente esencial para el modelado del negocio la presente propuesta de integración.

Los productos obtenidos para cada uno de los componentes de la arquitectura propuesta fueron los siguientes:

\subsection{Descripción del negocio e identificación del problema}

Mediante un conjunto de afirmaciones proporcionadas por 15 representantes del cliente junto con la correspondiente documentación que la respalda, fue posible obtener una descripción del negocio, los procesos que lleva a cabo y la identificación del problema. La tabla 5 muestra las afirmaciones proporcionadas por 2 representantes diferentes del cliente.

Tabla 5: afirmaciones proporcionadas por los representantes del cliente

\begin{abstract}
Afirmación 1. Representante de la Oficina de RDS: "La Facultad de Ciencias de la salud tiene a su cargo 4 programas: Medicina, Enfermería, Fisioterapia y Fonoaudiología. Los estudiantes de dichos programas deben realizar un procedimiento que implica que cada uno de ellos deba diligenciar su hoja de vida para poder ingresar a los escenarios de práctica, ellos requieren que los estudiantes estén a paz y salvo sobre vacunas, seguridad social, tener toda la documentación al día, etc. Las secretarias y los coordinadores de cada asignatura deben revisar esas hojas de vida y generar una lista de chequeo de los requisitos, además deben armar los cuadros de rotaciones y turnos, todo eso lo adjuntan en un CD y envían la información a mi oficina, Yo me encargo de despacharla a cada uno de los escenarios de práctica con una carta de presentación firmada por mí, ellos allá observan que todo esté en regla, y si es asi permitirán el ingreso a los estudiantes para finalmente llevar a cabo las prácticas". Documentos: modelo de Hoja de vida de estudiante, lista de verificación de requisitos de estudiantes, modelo del cuadro de rotes, modelo del cuadro de turnos. - RDS: Relación Docencia Servicio.
\end{abstract}

Afirmación 2. Representante de los Departamentos: "Los rotes se hacen de la siguiente manera: 1. Se definen las listas de estudiantes, 2. Se dividen a los estudiantes en grupos (dependiendo del número de docentes y estudiantes, la norma dice que deben ser MÁXIMO 5 estudiantes por grupo, pero en muchas ocasiones los grupos quedan de 6 estudiantes), 3. Se establecen las fechas de rotaciones, 4. Se envía la información de hojas de vida y grupos de rote a convenios (antes que los estudiantes vayan a rotar, pero no se da por no tener las listas a tiempo. Para los turnos: 1. Se debe disponer lista definitiva de estudiantes, 2. Distribuir estudiantes de VII y VIII (2 por dia) En los días de los meses que dura el se mestre. Nota: Para este proceso ya se han enviado las hojas de vida de estudiantes, no se deben enviar nuevamente". Documento(s): Cuadro de rotes diligenciado, Hojas de vida de algunos estudiantes, lista de verificación diligenciada, cuadro de turnos diligenciado, información adicional de soporte.

Fuente: elaboración propia 
La tabla 6 recoge las descripciones del negocio, procesos e identificación del problema.

Tabla 6: ficha de descripción del negocio, procesos e identificación del problema

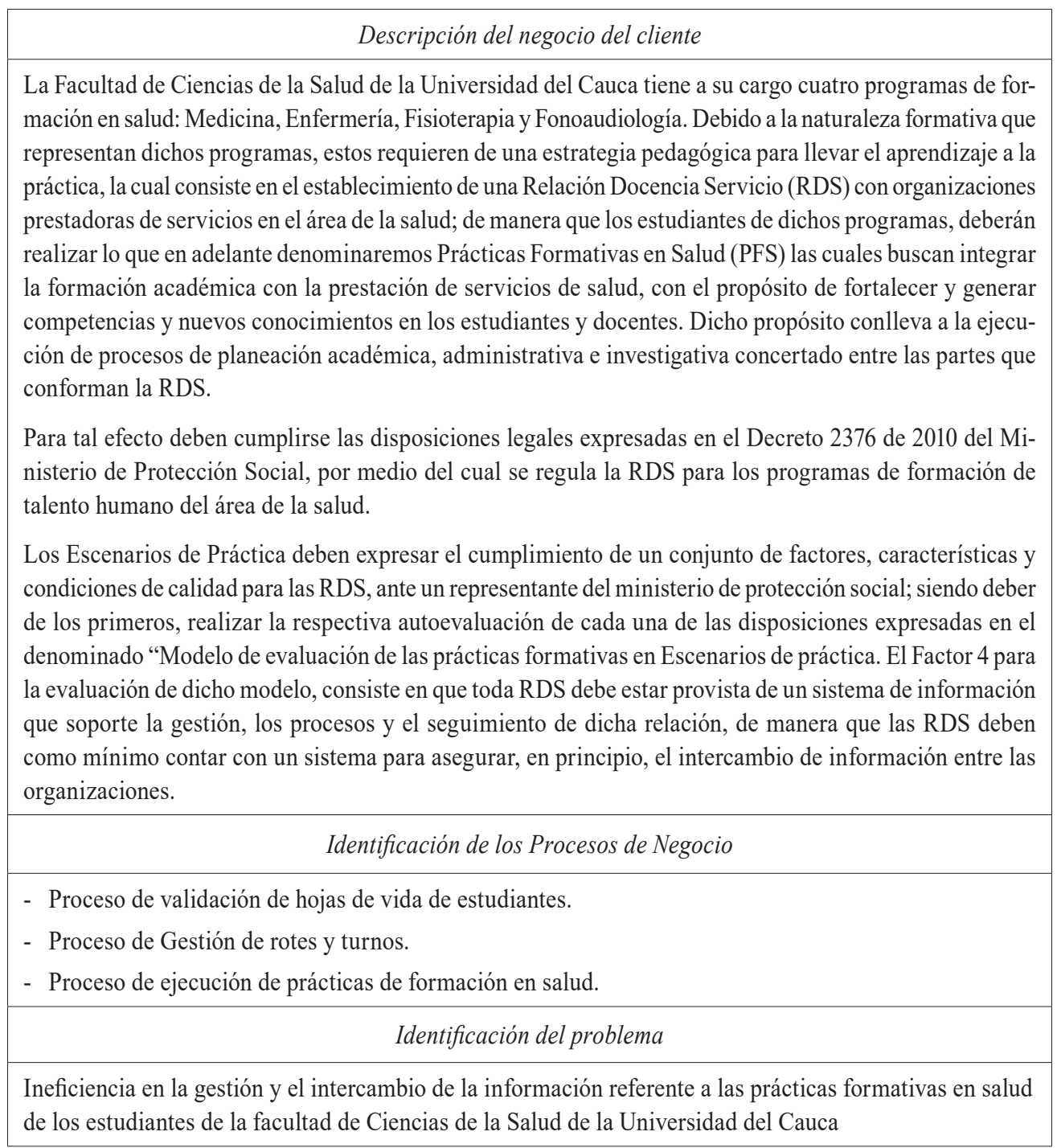

Fuente: elaboración propia

\subsection{Obtención del GN, ON y RN}

A partir de la información sintetizada en el apartado previo fueron constituidos los artefactos GN, ON y RN, descritos parcialmente en la tabla 7. 
Tabla 7: GN, ON y RN

Glosario del negocio (parcial)

Relación Docencia Servicio (RDS): estrategia pedagógica que establece una unión entre las instituciones educativas y organizaciones prestadoras de servicios en el área de la salud para la ejecución de las Prácticas Formativas en Salud (PFS).

Prácticas Formativas en Salud (PFS): son aquellas que buscan integrar la formación académica con la prestación de servicios de salud.

Decreto 2376 de 2010: Por medio del cual se regula la relación docencia - servicio para los programas de formación de talento humano del área de la salud.

Escenarios de Práctica (EP): son espacios en los cuales se desarrollan las PFS.

Jefe de la Oficina de Convenios (JOC): persona encargada de gestionar del lado de la institución todo lo referente a la formalización de convenios, en especial y para el caso, el despacho de la información proveniente de los departamentos.

Docente: es la persona encargada de asesorar al estudiante en las PFS.

Estudiante: es la persona que recibe orientación en las PFS.

Hoja de vida de estudiante: es el documento que corresponde a toda la información que el estudiante necesita hacer constar para ser apto de ingresar a una PFS.

Cuadro de Rotes: es el documento que informa sobre la disposición de espacio (lugar) y tiempo (horario) que un conjunto de estudiantes deberá cumplir en un determinado sitio de práctica. Éste relaciona las áreas o servicios en los que rotan los estudiantes.

SIMCA: Es la sigla del Sistema Integrado de Matrícula y Control Académico, en este caso es utilizado para consultar la lista de estudiantes matriculados.

Objetivo del negocio

Nombre: Tramitar información para la realización de Rotes y Turnos de los estudiantes de la Facultad de Ciencias de la Salud de la Universidad del Cauca hacia los Sitios de Práctica.

Descripción: Siempre que los estudiantes vayan a rotar deberá enviarse información confiable y completa a los Sitios de práctica para su posterior verificación y consulta. Finalmente los estudiantes deberán ejecutar sus respectivas PFS.

Reglas del negocio (parcial)

Referentes al Estudiante:

1. Todo estudiante está autorizado a proporcionar la información necesaria para la formalización de sus respectivos rotes y/o turnos, solo si está activo en SIMCA.

2. No puede ejecutarse ningún trámite que no posea el aval de la oficina de convenios

3. ....

Referentes al Departamento

1. Toda asignatura tiene que ser gestionada por 1 Departamento.

2. Todo estudiante tiene que ser gestionado por 1 Departamento.

3. ...

Referentes al Escenario de Práctica

1. Todo estudiante tiene que aprobar el curso de inducción al EP. 
2. Todo estudiante que este cursando un rote debería estar presente en ese rote.

3. ...

Referentes a la Oficina de Convenios

1. La Oficina de Convenios tiene que tener respaldo físico y actualizado de las pólizas de responsabilidad civil y riesgos biológicos.

2. ...

Fuente: elaboración propia

\subsection{LMN de la organización y derivación del MCUN y del MAN}

La figura 7 ilustra el LMN de la organización (modelo del negocio del cliente) obtenido con base en la información previa.

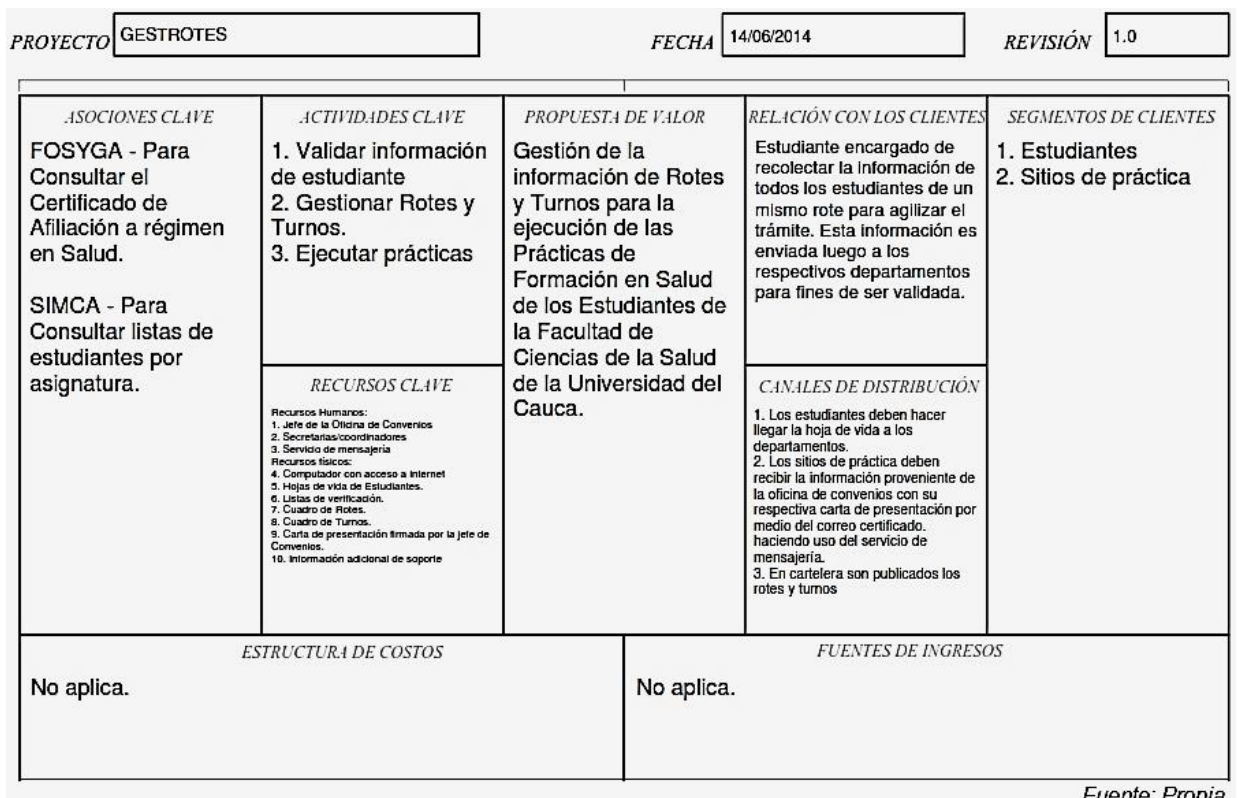

Figura 7: LMN para la descripción de la organización-cliente.

Fuente: elaboración propia

\subsection{Obtención del MCUN y el MAN}

A partir del LMN que describe la organización cliente, y aplicando la identificación de estereotipos y artefactos propuesta en el apartado 2.1.3 y en la figura 6, se derivan el MCUN y el MAN requeridoS por el MCS. La figura 8 y la figura 9 ilustran de manera parcial tales modelos. 


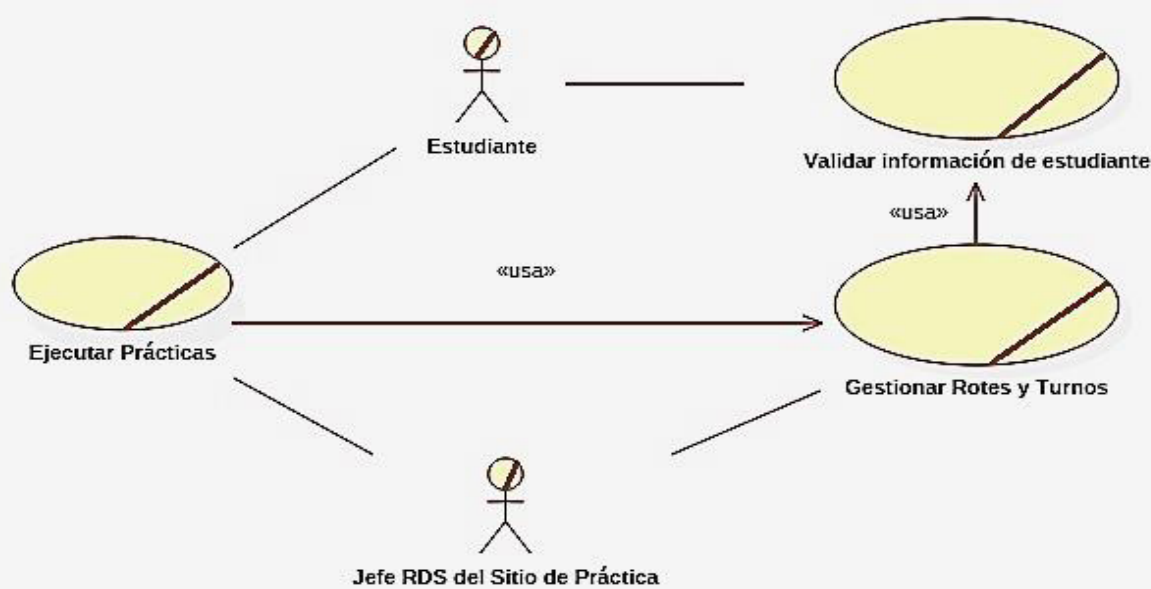

Figura 8: modelo de Casos de Uso del Negocio.

Fuente: elaboración propia

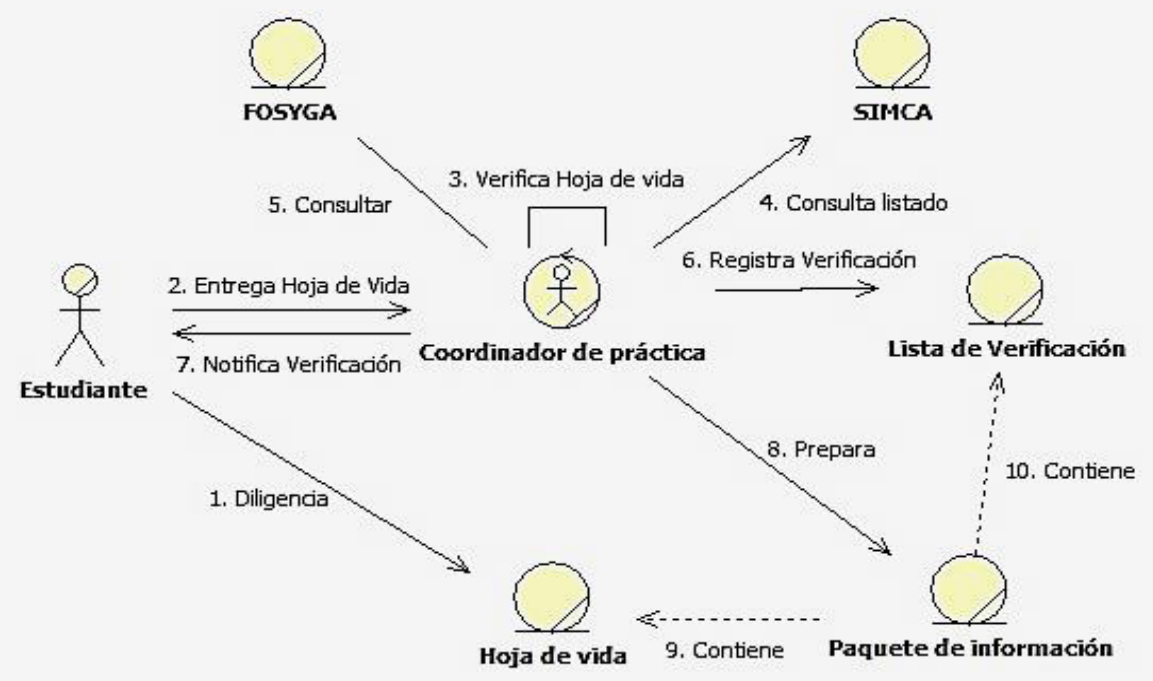

Figura 9: modelo de Objetos - Validar Información de Estudiante.

Fuente: elaboración propia

\subsection{LMN para la descripción del sistema / solución}

Con base en la información recabada y siguiendo las preguntas propuestas en el apartado 2.1.4 se obtiene el Lienzo del Modelo de Negocio del Sistema/Solución (ver figura 10). 


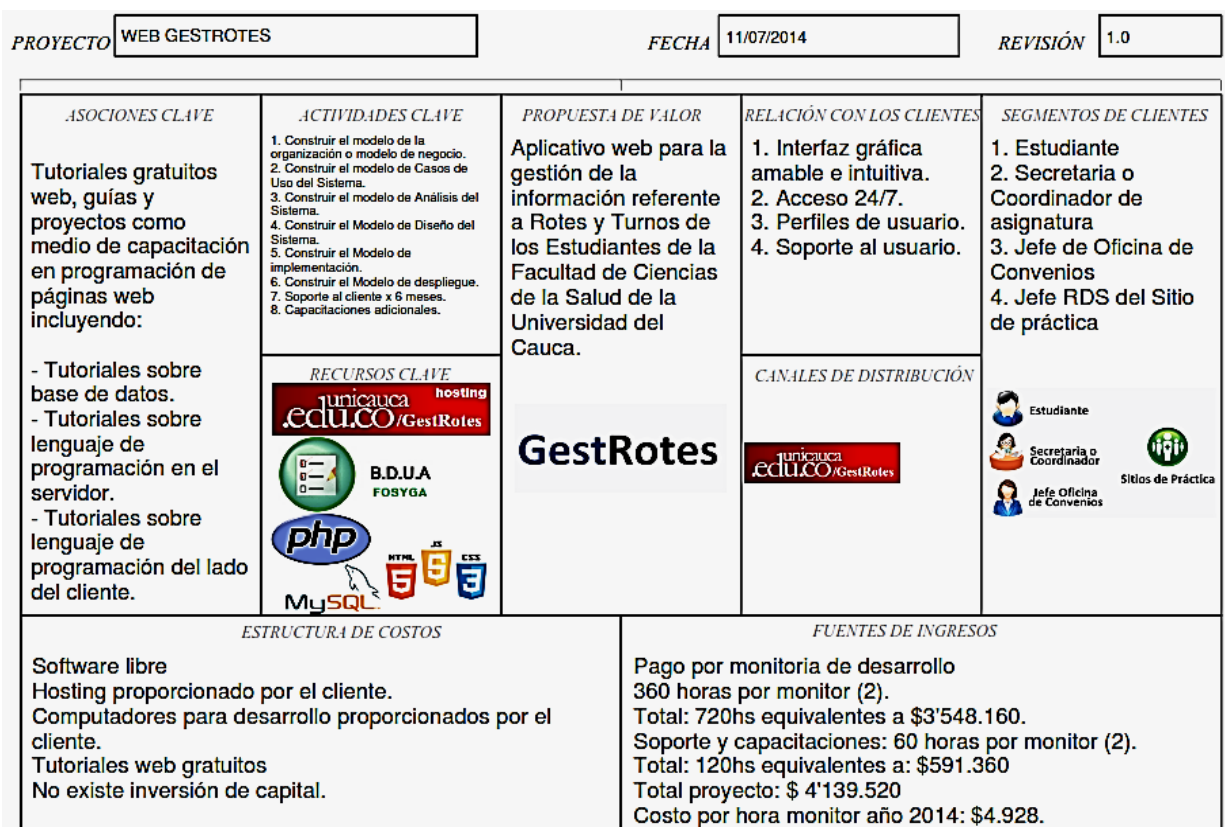

Figura 10: LMN para la descripción del Sistema/Solución.

Fuente: elaboración propia

El LMN que describe el sistema / solución constituye una herramienta que le provee al equipo de desarrollo una primera visión general de sistema a desarrollar, en un lenguaje que facilita el diálogo y negociación con el cliente. Asimismo, siguiendo los lineamientos de esta propuesta de integración, el LMN para la descripción del sistema / solución es una entrada desde la cual identificar los diversos componentes que faciliten construir el modelo de casos de uso y el modelo de análisis en UML para el sistema solución.

\subsection{Introducción de un PMV}

Para este ejercicio se incluyó un PMV para la comprobación de los siguientes objetivos:

1. Validar con el cliente el modelo de negocio y el sistema propuesto.

2. Dotar al cliente de una capacidad inicial sobre el sistema a construir.

Para el primer objetivo fue publicada una "landing page", vía correo electrónico, a los futuros usuarios del sistema, para la promoción de la página "GestRotes" la cual contiene un vídeo que describe el modelo de negocio, además del servicio que sería implementado. El vídeo puede verse en el enlace:

https://www.unicauca.edu.co/gestrotes/videos/queesgestrotesfinal.mp4 
Para el segundo objetivo fue seleccionado el procedimiento más común de "compartir información", de manera que fue implementada una interfaz simple para cada uno de los cuatro tipos de usuarios: coordinadores de práctica, jefe de oficina de convenios, sitio de práctica y estudiante. La única funcionalidad fue la de cargar información; el resto solo representaba una fachada de lo que podría implicar la lógica del sistema, como, por ejemplo, distribuir a los estudiantes según la hora y el lugar o enviar información a su destinatario, etc. La figura 11 muestra un ejemplo de la interfaz gráfica para el usuario "Departamento".

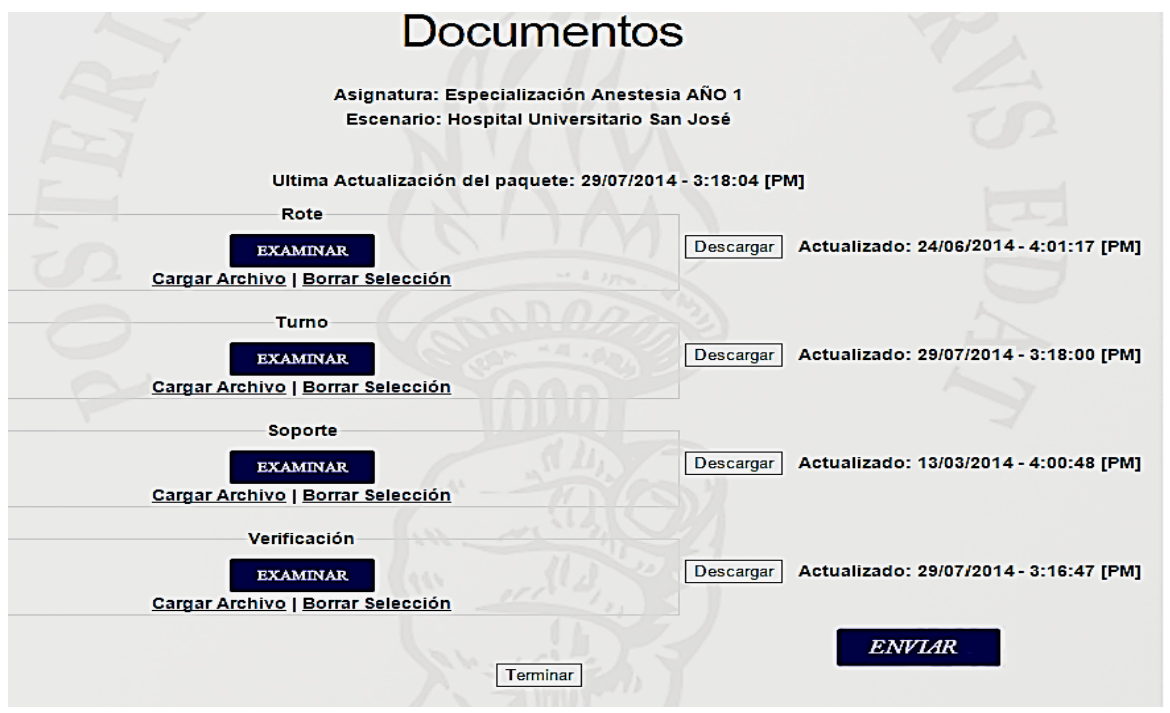

Figura 11: interfaz gráfica para el usuario "Departamento".

Fuente: elaboración propia

Con base en toda la información proporcionada al desarrollar cada una de las actividades del marco de referencia para la integración de MCS y el MNC, fue posible contribuir significativamente a la implementación del sistema / solución, debido al oportuno entendimiento del funcionamiento de la organización. En la actualidad "GestRotes" gestiona la información de más de 1300 estudiantes de cuatros programas, 53 asignaturas del área de formación en salud (fisioterapia, fonoaudiología, enfermería y medicina) y a 6 escenarios de práctica (hospitales o clínicas).

\section{CONCLUSIONES}

Cabe destacar que el presente trabajo de investigación constituye una aproximación inicial a la integración del concepto de modelo de negocio en sus diferentes connotaciones, tanto aquella que sirve para saber la mejor manera de ofrecer valor, como 
la que instruye a un equipo de desarrollo para el entendimiento de una determinada organización. Para contar con una evidencia más amplia sobre la bondad de esta propuesta de integración se hace necesario desarrollar trabajos adicionales que utilicen el marco de referencia expuesto. No obstante, es posible adelantar algunas conclusiones que este ejercicio inicial permite vislumbrar.

La arquitectura de trabajo propuesta constituye un marco de referencia para la integración del MNC con el MCS para el desarrollo de sistemas telemáticos, con impacto a lo largo del proceso de desarrollo, en especial en el estudio de prefactibilidad, ya que facilita el diálogo entre los interesados y la obtención del modelo de negocio requerido como paso inicial en el proceso de desarrollo del sistema / solución.

El marco de referencia propuesto complementa al MCS con artefactos que facilitan el trabajo inicial del analista del sistema para modelar la organización cliente, tales como el glosario del negocio, objetivos del negocio, reglas del negocio y el lienzo del modelo del negocio Canvas del cliente. Si bien en el MCS se plantea la necesidad de obtener los dos primeros, no se recomiendan formas que faciliten su desarrollo.

El modelo genérico para el modelado del negocio propuesto en [11] permitió articular los tres artefactos: glosario del negocio, objetivo del negocio y las reglas del negocio. Valga aclarar que el resto de los artefactos implicados cumple la función de brindar detalle explícito en caso de que se requiera. En este caso, los tres artefactos fueron razón suficiente y necesaria para comprender la lógica de la organización en cuestión.

Partir de las afirmaciones de los clientes para construir el modelo de negocio sirvió de gran ayuda en la descripción del modelo de negocio de nuestro caso de estudio. Sin embargo, clasificar adecuadamente la información es una regla implícita en el marco de trabajo propuesto.

\section{AGRADECIMIENTOS}

Los autores agradecen a la Secretaría General de Facultad de Ciencias de la Salud de la Universidad del Cauca por permitirnos emplear, por primera vez, el marco de referencia para la obtención del modelo de negocio para el desarrollo de la plataforma GestRotes.

\section{REFERENCIAS}

[1] C. Serrano, M. Solarte y G. Ramirez, Referencia Metodológica Integral para el Desarrollo de Sistemas Telemáticos, Popayán, Cauca: Universidad del Cauca, 2004, p. 98.

[2] A. Osterwalder y Y. Pigneur, Generación de modelos de negocio, 1 ed., Barcelona: Deusto, 2011, p. 285. 
[3] B. Baker, Business Modeling with UML: The Light at the End of the Tunnel, Copyright Rational Software, 2001, p. 9.

[4] J. Montilva, "Modelado de Negocios "Del espacio del problema al espacio de la solución"”, de IDEAS'07 I Workshop Iberoamericano de Ingeniería de Requisitos y Ambientes de Software, Mérida, 2007.

[5] G. Booch, I. Jacobson y J. Rumbaugh, El proceso unificado de desarrollo de software, Madrid: Addison-Wesley, 2006, p. 464.

[6] G. Booch, J. Rumbaugh y I. Jacobson, The Unified Modeling Language User Guide, Boston: Addison-Wesley, 2005, p. 496.

[7] A. Osterwalder, “The Business model Ontology,” Lausanne, 2004.

[8] A. Osterwalder y Y. Pigneur, Value Proposition Design, New Jersey: Wiley, 2014.

[9] E. Ries, El método Lean StartUp "Cómo crear empresas de éxito utilizando la innovación contínua”, 5 ed., Barcelona: Deusto, 2011, p. 320.

[10] S. Blank, The Four Steps to the Epiphany, 2nd ed., K\&S Ranch, 2013, p. 370.

[11] EXECyL, Emprendedor: Qué debes saber sobre Lean StartUp, 2014, p. 43.

[12] G. Baum, M. Daniele, P. Martellotto y M. Novaira, Un modelo Genérico para el Modelo de Negocio, Río Cuarto, Córdoba, 2004. 